A tapering window for time-domain templates and simulated signals in the detection of gravitational waves from coalescing compact binaries

This article has been downloaded from IOPscience. Please scroll down to see the full text article.

2010 Class. Quantum Grav. 27084020

(http://iopscience.iop.org/0264-9381/27/8/084020)

View the table of contents for this issue, or go to the journal homepage for more

Download details:

IP Address: 131.251.133.26

The article was downloaded on 04/04/2012 at 12:43

Please note that terms and conditions apply. 


\title{
A tapering window for time-domain templates and simulated signals in the detection of gravitational waves from coalescing compact binaries
}

\author{
D J A McKechan, C Robinson and B S Sathyaprakash \\ Queens Buildings, The Parade, Cardiff, CF24 3AA, UK \\ E-mail: david.mckechan@astro.cf.ac.uk, craig.robinson@astro.cf.ac.uk and \\ b.sathyaprakash@astro.cf.ac.uk
}

Received 12 November 2009, in final form 18 January 2010

Published 6 April 2010

Online at stacks.iop.org/CQG/27/084020

\begin{abstract}
Inspiral signals from binary black holes, in particular those with masses in the range $10 M_{\odot} \lesssim M \lesssim 1000 M_{\odot}$, may last for only a few cycles within a detector's most sensitive frequency band. The spectrum of a square-windowed time-domain signal could contain unwanted power that can cause problems in gravitational wave data analysis, particularly when the waveforms are of short duration. There may be leakage of power into frequency bins where no such power is expected, causing an excess of false alarms. We present a method of tapering the time-domain waveforms that significantly reduces unwanted leakage of power, leading to a spectrum that agrees very well with that of a long duration signal. Our tapered window also decreases the false alarms caused by instrumental and environmental transients that are picked up by templates with spurious signal power. The suppression of background is an important goal in noise-dominated searches and can lead to an improvement in the detection efficiency of the search algorithms.
\end{abstract}

PACS numbers: $02.30 . \mathrm{Nw}, 04.30 .-\mathrm{w}, 04.80 . \mathrm{Nn}$

(Some figures in this article are in colour only in the electronic version)

\section{Introduction}

Interferometric gravitational wave detectors are now operating at sensitivity levels at which one can expect to detect inspirals from compact binary coalescences at the rate of one per 5 years (optimistic rate) to one per 5000 years (pessimistic rate). When upgraded to advanced detectors, these might be as large as 400 per year to one per 2.5 years [1]. Even so, most of the inspiral signals are not likely to stand above the noise background. A variety of techniques to enhance signal visibility and reject false alarms are currently being used in gravitational wave searches. Examples include matched filtering for signals of known phase evolution [2], 
wavelet transforms for transient signals of unknown shape [3, 4], coherent search methods for burst signals [5], etc. Moreover, vetoes based on the expected signal evolution [6] and instrumental and environmental monitors [7] have been developed over the past decade to improve detection probability and mitigate false alarms. Detecting a signal buried in nonstationary noise is a challenging problem as some types of non-stationary noise artefacts can partially mimic the signal.

Many of these techniques involve the computation of a correlation integral in which bandpassed data are multiplied by the FD model waveform or the DFT of the TD signal (see, for example, [8]). Here we consider a matched filtering search for inspiral signals where the DFT of a TD waveform is used to construct the correlation. A problem that has not been adequately addressed (see, however, [9]) in this context is the effect of the window that is used in chopping a TD signal before computing its DFT.

Inevitably, all signal analysis algorithms use, implicitly or explicitly, some form of window function. An inspiral waveform sampled from a time when the signal's instantaneous frequency enters a detector's sensitive band until the time when it reaches the FLSO implicitly makes use of a square window. Signal analysis literature is full of examples of artefacts caused by the use of such window functions. For instance: leakage of power from the main frequency bin where the signal is expected to lie into neighbouring bins, loss of frequency resolution and corruption of parameter estimation [10]. In this paper we explore the problems caused by using a square window and suggest an alternative that cures some of the problems.

There is no unique, or favoured, windowing method. One is often guided by the requirements of a particular analysis at hand. In our case, a square window is especially bad since the leakage of power outside the frequency range of interest can lead to increased false alarm rate and poorer estimation of parameters. One reason for increased false alarm rate could be that the noise glitches in the detector look more like the untapered waveform and less like a tapered one. We have explored the effect of a smoother window function, presented in section 2, which has a far steeper fall-off of power outside the frequency range of interest. Use of this window has cured several problems we had with a square window. In section 4 we will discuss how tapering helps in a more reliable signal spectral estimation and hence a proper determination of the expected signal-to-noise ratio. Spectral contamination is worse for larger mass black hole binaries as they are in the detector's sensitive band for a shorter time and the window function can only extend over a short time. It is for such signals that our tapered window offers the most improvement. In section 5 we will discuss how the rate of triggers from a matched filtered search can vary depending on the kind of window function used. We shall briefly mention in section 6 what effect our window function has on parameter estimation, giving the conclusions of our study in section 7 .

\section{Window functions and their temporal and spectral characteristics}

Let $h(t)$ denote a continuous differentiable function, for example a gravitational wave signal emitted by a coalescing compact binary, and let $H(f)$ denote the FT of $h(t)$ defined by

$$
H(f) \equiv \int_{-\infty}^{\infty} h(t) \exp (2 \pi \mathrm{i} f t) \mathrm{d} t
$$

In reality the signal really does not last for an infinite time. The FT of a signal of finite duration lasting, say, from $-T / 2$ to $T / 2$, can be represented either by using the limits of the integral to go from $-T / 2$ to $T / 2$ or by using a window function. The latter is preferred so as to preserve the definition of the FT. 
A window function is a function that has either a finite support or falls off sufficiently rapidly as $t \rightarrow \pm \infty$. Two simple windows that have finite support are the square window $s_{T}(t)$ defined by

$$
s_{T}(t)= \begin{cases}1, & -\frac{T}{2} \leqslant t \leqslant \frac{T}{2} \\ 0, & \text { otherwise }\end{cases}
$$

and the triangular window $b_{T}(t)$ defined by

$$
b_{T}(t)=\left\{\begin{array}{lc}
(1-2|t| / T), & -\frac{T}{2} \leqslant t \leqslant \frac{T}{2} \\
0, & \text { otherwise. }
\end{array}\right.
$$

Neither the square nor the triangular window are differentiable everywhere. As a result, they are not functions of finite bandwidth. In other words, their FTs, $S_{T}(f)$ and $B_{T}(f)$, do not have finite support in the FD: $|S(f)|>0$ for $-\infty \leqslant f \leqslant \infty$. In the case of a square window the FT $S(f)$ is a sinc function, $\left|S_{T}(f)\right|=T \operatorname{sinc}(\pi f T)$, which is peaked at $f=0$, with a width $\pi / T$ and falls off as $f^{-1}$ as $f \rightarrow \pm \infty$. The lack of finite support in the Fourier domain could sometimes cause problems, especially when the width of the window in TD is too small. For functions that have infinite bandwidth the sampling theorem does not hold but this is not a serious drawback if the FT falls off sufficiently fast above the Nyquist frequency. However, there could be other issues when the window leads to leakage of power outside a region of interest as we shall see below.

\subsection{The Planck-taper window function}

A signal $h(t)$ with the window $w_{T}(t)$ applied to it, in other words the windowed signal $h_{w}(t)$, is defined by

$$
h_{w}(t) \equiv h(t) w_{T}(t)
$$

The convolution theorem states that the FT of the product of two functions $h(t)$ and $w_{T}(t)$ is the convolution of individual FTs:

$$
\begin{aligned}
H_{w}(f) & =\int_{-\infty}^{\infty} h(t) w_{T}(t) \exp (2 \pi \mathrm{i} f t) \mathrm{d} t \\
& =H(f) * W_{T}(f)=\int_{-\infty}^{\infty} H\left(f^{\prime}\right) W_{T}\left(f-f^{\prime}\right) \mathrm{d} f^{\prime}
\end{aligned}
$$

We can now see why a window whose power in the FD does not fall off sufficiently rapidly might be problematic. The convolution integral will have contributions from all frequencies. Suppose we are interested in matched filtering the data with an inspiral signal from a compact coalescing binary whose instantaneous frequency varies from $f_{a}$ at time $t_{a}$ to $f_{b}$ at time $t_{b}$. One would normally achieve this by using a square window $s_{T}(t)$ that is centered at $\left(t_{a}+t_{b}\right) / 2$ with width $T=t_{b}-t_{a}$. However, we can see from (6) that the convolution integral will have contributions from outside the frequency range of interest.

To circumvent this problem we propose to use a window function that falls off rapidly outside the frequency range of interest. Inspired by the tapering function used in 

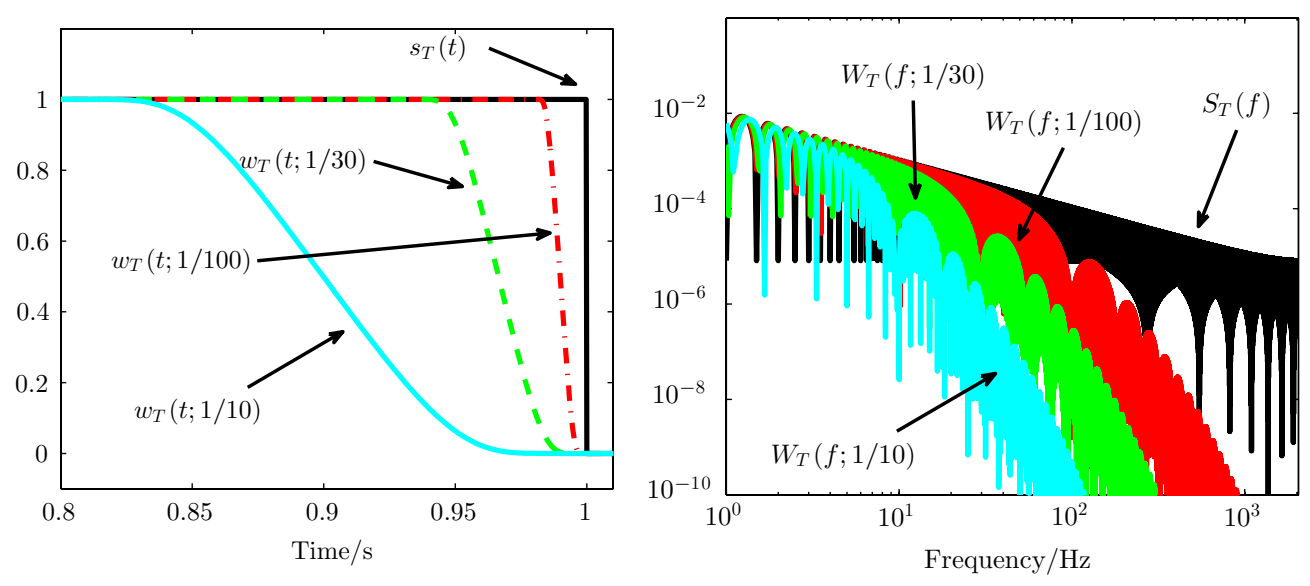

Figure 1. The Planck-taper window in the TD (left), for three different choices of the parameter $\epsilon=0.01,0.033,0.1$, and their power spectra (right). For reference we have included the square window with the same effective width as the Planck-taper window.

Damour et al [11] we define a new window function $\sigma(t)$ by

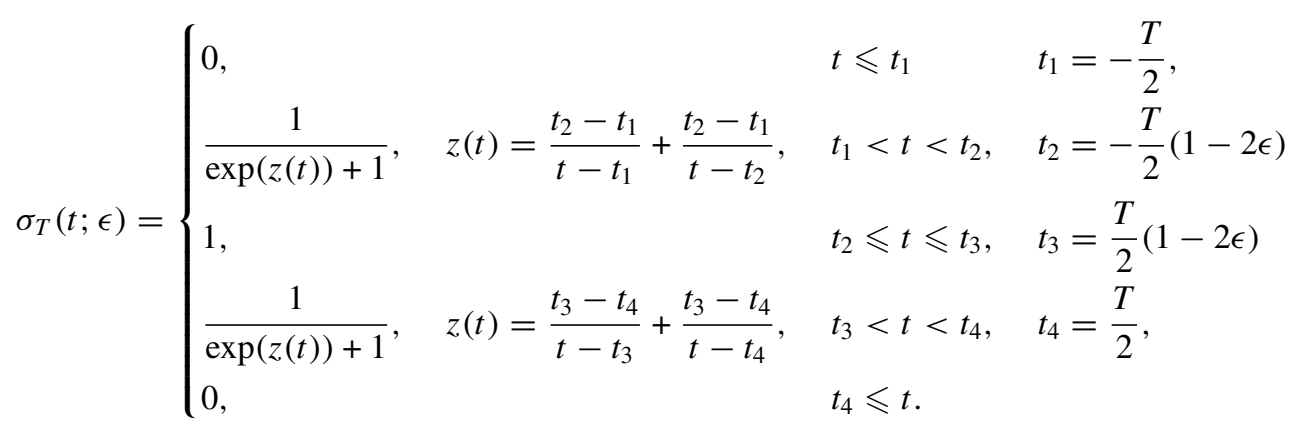

Here $T$ is the width of the window and $\epsilon$ is the fraction of the window width over which the window function smoothly rises from 0 at $t=t_{1}$ to 1 at $t=t_{2}$ or falls from 1 at $t=t_{3}$ to 0 at $t=t_{4}$. We shall call $\sigma(t)$ the Planck-taper window as the basic functional form is that of the Planck distribution. The motivation for choosing this window function is to reduce the leakage of power in the FD but at the same time not to lose too much of the length of the signal in the TD. The choice of $\epsilon$ will affect both aspects significantly. In figure 1 we have shown the window function for several choices of the parameter $\epsilon=0.01,0.033,0.1$. At lower frequencies the spectrum of the Planck-taper window falls off at the same rate (i.e. $1 / f$ ) as a square window. But beyond a certain frequency $f_{0} \sim(\epsilon T)^{-1}$, the spectrum falls off far faster.

A key feature in our use of the Planck-taper window is the automated and waveformdependent adjustment of $\epsilon$ as discussed in section 2.2 below.

\subsection{Implementation of the window}

We discretize (7) by replacing $t, t_{1}, t_{2}, t_{3}, t_{4}$ with the array indices $j, j_{1}, j_{2}, j_{3}, j_{4}$. In this notation the parameter epsilon is approximated by $\epsilon \simeq\left(j_{2}-j_{1}\right) / N$, where $N$ is the number 


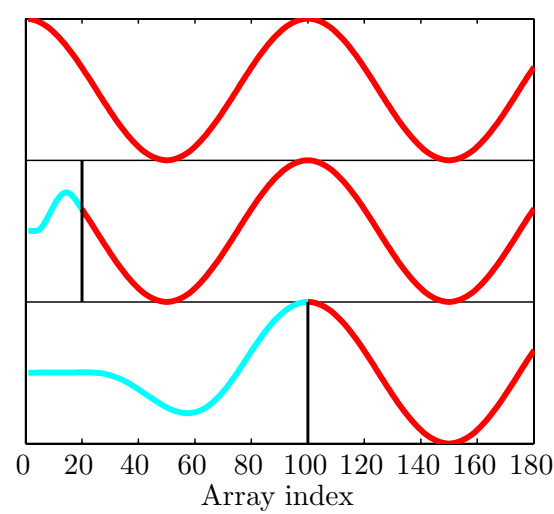

Figure 2. The window function has been applied to the start of a cosine wave (top curve) using two methods. In the first case it is applied from $j=1$ up to an arbitrary choice of $j=20$ (middle), whereas in the second case it is applied up to the second maximum at $j=100$ (bottom). The lighter coloured parts of the middle and bottom curves (to the left of the black vertical lines) show where the taper has been applied.

of data points in the waveform. The start and end of the waveform are denoted by $j_{1}$ and $j_{4}$, respectively. The values of $j_{2}$ and $j_{3}$ have to be chosen judiciously to avoid leakage of power. We choose $j_{2}$ and $j_{3}$ to be the array index corresponding to the second stationary point after $j_{1}$ and before $j_{4}$ (see figure 2). Applying the transition stage of $\sigma$ from a crest/trough ensures that the window does not have a sudden impact on the behaviour of the waveform. The first stationary point would not be an appropriate choice as it may occur within only a few array points of $j_{1}$ or $j_{4}$, causing $\epsilon$ to be too small. One could choose the 3 rd, 4 th or 5 th, but using such later maxima would reduce the genuine power of the waveform more than what might be acceptable.

\subsection{Comparison with other windows}

We do not compare the performance of Planck taper with other commonly used windows, e.g., Bartlet, Hann or Welch. Such windows transition between 0 and 1 over $j=1, \ldots, N / 2$, where the window is of length $N$, producing significant differences between $h(t)$ and $h_{w}(t)$ in (4). The power is therefore suppressed at the beginning and end of $h(t)$. This is acceptable when computing the PSD of a data segment, but would cause a problem if applied to a template waveform as the phase (frequency) and amplitude of $h(t)$ are both instantaneous functions of $t$, with the most power at the end of the waveform.

Windows with properties similar to Planck taper, such as having a central flat region, do exist. For example, the Tukey window [12], which has been used in gravitational-wave data analysis recently [13], may offer a good comparison. However, a key feature in our study of the Planck-taper window is the waveform-dependent adjustment of $j_{2}$ and $j_{3}$. Whilst this automation could be considered separately from the Planck-taper window and used on other windows defined by the points $j_{1,2,3,4}$, we have not done so here. Given the shared features of the Tukey window with Planck taper one might expect similar results.

\section{Effect of the window function on the signal spectrum}

In this section we will examine the power spectrum of the waveform of a coalescing binary emitted during the inspiral phase. The waveforms are modelled using the PN approximation. 
$20 M_{\odot}$

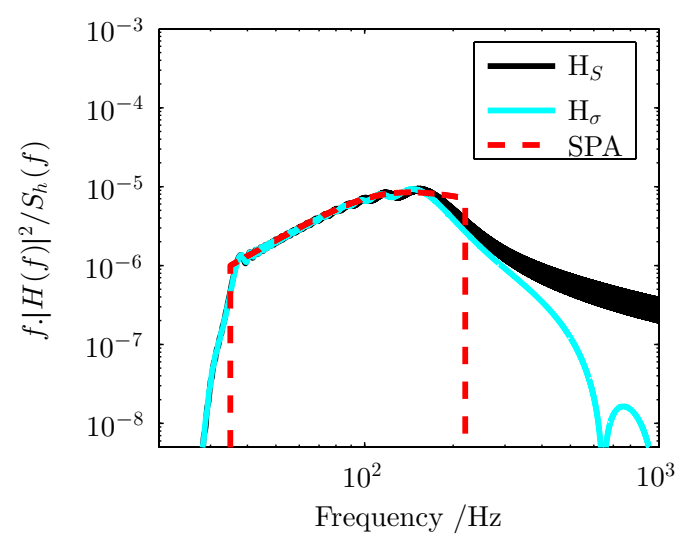

$80 M_{\odot}$

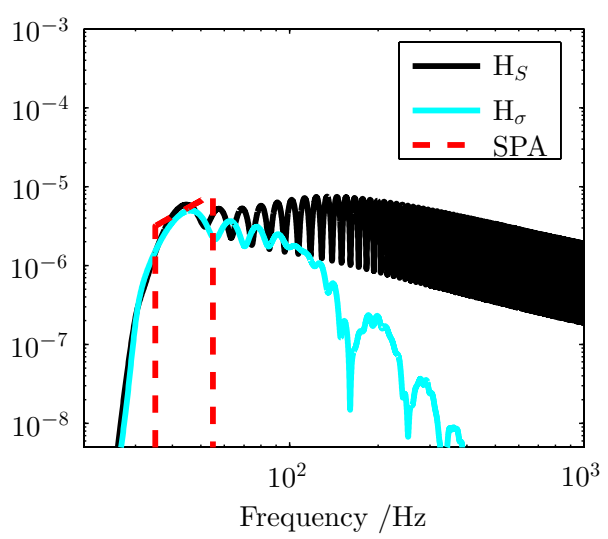

Figure 3. The plots show the SNR integrand, where the waveform is generated from a frequency of $35 \mathrm{~Hz}$ to the FLSO of the source, computed using the initial LIGO sensitivity for sources of total mass $20 M_{\odot}$ and $80 M_{\odot}$. In the case of the Planck-taper window the SNR integrand falls off far faster than in the case of the square window.

However, even within the PN approximation, there are several different ways in which one might construct the waveform $[14,15]$. Two such models widely used in the search for compact binary coalescences are TT3 and the SPA. TT3 is a TD signal model in which the amplitude and phase of the signal are both explicit functions of time. In the so-called restricted PN approximation the signal consists of the dominant harmonic at twice the orbital frequency, but not higher order PN corrections consisting of other harmonics, and the phase is a PN expansion that is currently known to $\mathcal{O}\left(v^{7}\right)$ in the expansion parameter $v$-the relative velocity of the two stars. The SPA is the Fourier transform of the TT3 model obtained by using the stationary phase approximation to the Fourier integral [16]. A template belonging to the TT3 model is defined for times when the gravitational wave frequency is within the detector's sensitivity band until it reaches FLSO. This means one is in effect multiplying a square window with a continuous function.

Figure 3 shows the SNR integrand of the SPA, computed using the initial LIGO design PSD [14]. The inspiral waveform is defined from a lower cutoff frequency of $35 \mathrm{~Hz}$ up to its FLSO, for $20 M_{\odot}$ and $80 M_{\odot}$ equal-mass binaries. The DFT of the TT3, generated between the same frequencies, with a square window (or rather no window), labelled $\mathrm{H}_{S}$, and with the Planck-taper window, labelled $\mathrm{H}_{\sigma}$, are also plotted. Where the Planck-taper window is used the excess power, that above FLSO, decreases rapidly and the spectrum is closer to that of the SPA.

\section{Effect of the window function on the estimation of the signal-to-noise ratio}

Gravitational wave searches for known signals, such as those emitted from a CBC [17, 18], rely upon signal models for two primary reasons. Firstly, they are used as templates to matched filter the data. Secondly, they are injected into the data as simulated signals to estimate the efficiency of the detector to detect such signals. If the signal/template models are generated in the TD then they must undergo a DFT if the data are analysed in the FD as is the case for the current LIGO matched filter code. 


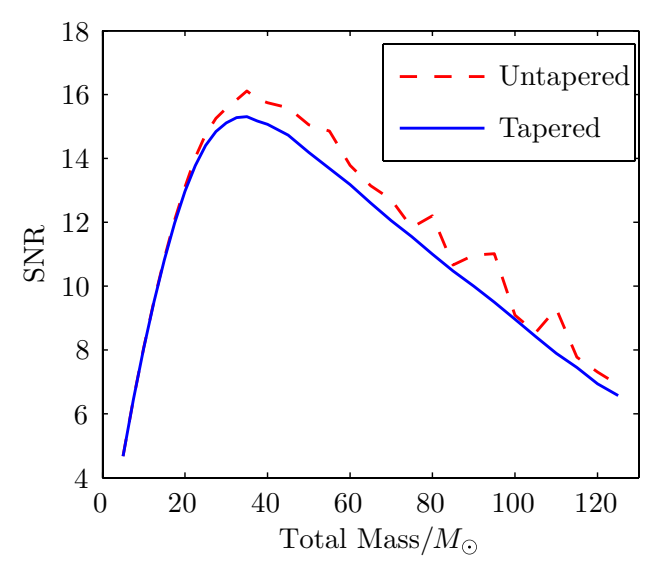

Figure 4. The SNR versus the total mass of the source for signals corresponding to compact binary systems directly overhead a detector of initial LIGO design PSD. We plot the SNR obtained using the DFT of TD waveforms with a square window (dashed curve) and with the Planck-taper window (solid curve). Here the systems are overhead the detector at an effective distance of $65 \mathrm{Mpc}$, using a fixed mass ratio of 5:1 and a fixed inclination angle of $45^{\circ}$.

The expectation value for the SNR of a signal in stationary Gaussian noise, when the signal and template match exactly, is given by

$$
\left\langle\rho^{2}\right\rangle=4 \int_{f_{\text {lower }}}^{f_{\text {nyquist }}} d f \frac{|H(f)|^{2}}{S_{h}(f)} \simeq 4 \Delta f \sum_{k=1}^{N / 2-1} \frac{\left|H_{k}\right|^{2}}{S_{h k}},
$$

where $S_{h}(f)$ is the detector noise PSD and $f_{\text {lower }}$ is the detector lower cutoff frequency chosen so that the contribution to the SNR integral from frequencies $f<f_{\text {lower }}$ is negligible. The second of the expressions on the RHS is a discretized evaluation of the SNR which is often used in numerical calculations. Here $H_{k}, k=0, \ldots, N / 2$, is the DFT of the signal defined for positive frequencies and $S_{h k}$ is the discretized PSD.

The amplitude of an inspiral signal increases with the total mass of the system; conversely, the FLSO of the signal is inversely proportional to the total mass. Therefore, as the total mass of a system increases, the amplitude of the signal and the FLSO will have opposing effects. For lower mass systems, the increasing amplitude causes the SNR to increase as a function of the total mass. However, for higher mass systems, the reduction in the FLSO causes the signal to have less power in band. As a result, the SNR will decrease as a function of the total mass. The relatively low FLSO of the higher mass templates, coupled with their short duration, lead them to be particularly susceptible to artefacts of spectral leakage in the DFT.

Figure 4 shows the SNR for TT3 inspiral waveforms that are 2PN in amplitude and phase, plotted as a function of the total mass for two choices of the window function: the dashed curve corresponds to the square window and the solid curve to the Planck-taper window. All other parameters are the same in both cases. When the Planck-taper window is used, the curve exhibits the expected behaviour, whereas in the case of a square window, the SNR curve is 'jagged' which is unexpected given that stationary Gaussian noise was used in the estimation of the SNR. This behaviour is most likely explained by the excess power from the DFT of the waveform.

It should be noted that integrating to FLSO rather than Nyquist in equation (8) is not considered appropriate here. Firstly, the higher harmonics in the amplitude corrected 
waveforms contain power above FLSO, (which becomes more significant for high mass systems). Secondly, cutting off the integration at FLSO is essentially the application of a square window to the template waveform in the frequency domain. This will lead to leakage of power in the time domain which is not a desirable feature. The problem of using a squarewindowed TD template as our matched filter is not that there is power above FLSO; it is that the excess power in this region, present due to windowing, but not present in a genuine signal will lead to unnecessary false alarms in a search.

\section{Effect of window functions on trigger rates}

To assess the effect that tapering of templates has on trigger rates, we have applied the LSC CBC pipeline [17-21] to data taken during the S4 of the LIGO, which took place from February 22-March 23, 2005. The basic topology of the pipeline is similar to that used in many previous searches $[17,18,20]$, and consists of the following main steps.

- The template bank is chosen such that the loss of SNR due to having a finite number of templates is no more than $3 \%$ for any signal belonging to a given family of waveforms $[2,22]$.

- Matched filter the data with the generated templates. A trigger is generated at times when the SNR is larger than a given threshold. The output of this stage is a list of first-stage single-detector triggers.

- Check for coincident events between different detectors. For an event to be deemed coincident, the parameters seen in at least two detectors (for instance, the masses of the system, the time of coalescence, etc) should agree to within a certain tolerance [23]. The output of this stage is a list of first-stage coincident triggers.

- Re-filter the data using only templates associated with coincident triggers. This time, the triggers are subjected to further signal-based vetoes, some of which are computationally costly, such as the chi-squared veto [6]. This produces a list of second-stage singledetector triggers.

- Check for coincident events between detectors using the second-stage single-detector triggers. This produces a list of second-stage coincident triggers.

In this study the data were filtered using the EOB templates [14, 24, 25], tuned to recent results in numerical relativity $[26,27]$, with a total mass in the range $25-100 M_{\odot}$. This choice agrees with the templates used to search for signals from high-mass CBCs in data from LIGO's S5. Because the EOB waveforms used as templates contain the inspiral, merger and ringdown phases, there was no need to taper the end of the waveform. Therefore, in this case, the taper specified in (7) was only applied to the start of the waveform. Although this may reduce the effect the taper has in comparison to tapering both ends of an inspiral-only template, it is of more interest to evaluate the performance in a realistic search case. It should be noted that the tapering window is explicitly applied to the template waveform where the length of the waveform is less than the length of the data segment that is matched filtered. We do not apply any window to the data segment.

Figure 5 shows the number of triggers as a function of total mass with and without tapering. It can be seen that the number of triggers is generally higher when the templates are not tapered. The only exception seems to be the lowest mass bin in the second-stage coincident triggers, where the opposite is true. However, the difference in the number of triggers in this bin is not large, and is likely just a statistical anomaly. For first-stage single-detector triggers, the number of triggers using tapered templates is $84 \%$ of that obtained using untapered templates. The number of second-stage coincident triggers when using tapered templates is $71 \%$ of that 

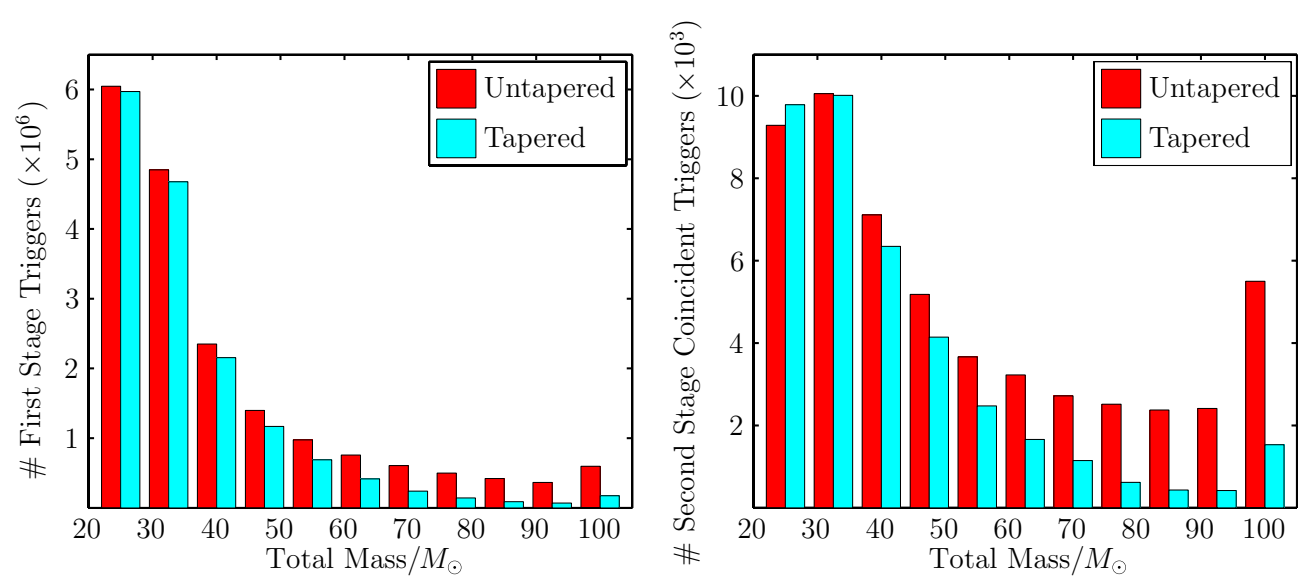

Figure 5. Left. Number of triggers recovered by matched filtering the S4 data with and without tapering applied to the templates. Right. Number of triggers at the second stage of the analysis pipeline, after consistency checks and coincidence tests [23] in the time-of-arrival and masses of the component stars have been applied.

obtained for untapered templates. The difference in trigger rates is more significant at higher masses. This is because the template waveforms for these systems terminate at a frequency within or below the most sensitive frequency band of the detector, making any leakage of power to higher frequencies more significant (cf figure 3, left most panel). The reduced trigger rate indicates that applying the taper function to the templates could aid in reducing the false alarm rate in a search for high mass CBCs.

\section{Effect of windowing on detection efficiency and parameter estimation}

The same data used in section 5 were reanalysed, but with simulated gravitational wave signals (injections) added. The injections were of the same family as the templates used in section 5. This allowed us to compare the detection efficiencies and accuracy of parameter estimation using tapered versus untapered templates. We looked at the error in recovered chirp mass and arrival time at both single-detector first-stage triggers and coincident second-stage triggers, but found negligible difference between the two cases ${ }^{1}$.

We did not explicitly measure the detection efficiency as a function of distance, but found the number of injections recovered to be nearly identical in the two cases, with less than $1 \%$ fewer injections found when using tapered templates. Given the vast reduction in the trigger rates shown in section 5, this indicates that an improvement in detection efficiency can be expected when using tapered templates.

The above studies were performed first with tapering applied to the injections and then repeated without - the difference between the results was negligible.

\section{Conclusion}

We have developed a tapering method that leads to a spectrum for TD waveforms that more closely matches their FD analogues, containing significantly less power at unexpected

1 We have seen some evidence of improvements in parameter estimation for the ambiguity function of high mass inspiral-only waveforms, but this is outside the context of a gravitational wave search. 
frequencies when compared with the use of a square window. This is achieved by automating the implementation of the window.

If tapering is applied to templates in a gravitational wave search the trigger rates are reduced, especially for high mass templates, without any significant change in detection efficiency. In a search, foreground triggers can be ranked by their probability of occurring as a background trigger; thus if background triggers are reduced, a given foreground trigger may appear more significant. Another benefit of reduced trigger rates is that the computational cost of a search will decrease. We have demonstrated that the windowing method would be beneficial when used in a high mass search.

The tapering method could also be useful in low latency data analysis techniques where TD templates are divided into sub-templates of different frequency ranges, and matched filtered individually [28]. The relative shortness of some templates in the higher frequency bands potentially compounds the problem of using a square window, and tapering the templates may go some way to alleviating this issue.

\section{Acknowledgments}

We thank the LSC for their permission to use the S4 data, without which this investigation would not have been possible. We would also like to thank Evan Ochsner and Anand Sengupta for their efforts in some of the initial tapering studies and the members of the CBC search group of the LSC-Virgo collaboration for allowing us to use the CBC search pipeline. We are grateful to Jolien Creighton for his critical analysis, which allowed us to clarify and improve this paper. We also thank Thomas Dent for carefully reading the manuscript and for his helpful suggestions. This work was funded in part by STFC studentship PPA/S/S/2006/4330 and STFC grant PP/F001096/1.

\section{References}

[1] Abadie J et al (LIGO Scientific Collaboration) 2010 arXiv:1003.2480

[2] Babak S, Balasubramanian R, Churches D, Cokelaer T and Sathyaprakash B 2006 Class. Quantum Grav. 23 5477-504 (arXiv:gr-qc/0604037)

[3] Klimenko S and Mitselmakher G 2004 Class. Quantum Grav. 21 S1819-30

[4] Klimenko S, Yakushin I, Rakhmanov M and Mitselmakher G 2004 Class. Quantum Grav. 21 S1685-94

[5] Chatterji S et al 2006 Phys. Rev. D 74082005 (arXiv:gr-qc/0605002)

[6] Allen B 2005 Phys. Rev. D 71062001

[7] Hanna C R (LIGO Scientific Collaboration) 2006 Class. Quantum Grav. 23 17-22

[8] Abbott B et al (LIGO Scientific Collaboration) 2004 Phys. Rev. D 69122001 (arXiv:gr-qc/0308069)

[9] Arnaud K A et al 2007 Class. Quantum Grav. 24551 (arXiv:gr-qc/0701170)

[10] National I 2009 Windowing: optimizing FFTs using window functions http://zone.ni.com/devzone/cda/tut/p/ $\mathrm{id} / 4844$

[11] Damour T, Iyer B R and Sathyaprakash B S 2000 Phys. Rev. D 62084036 (arXiv:gr-qc/0001023)

[12] Harris F J 1978 Proc. IEEE 66 51-83

[13] Röver C, Meyer R and Christensen N 2007 Phys. Rev. D 75062004 (arXiv:gr-qc/0609131)

[14] Damour T, Iyer B R and Sathyaprakash B S 2001 Phys. Rev. D 63044023

[15] Buonanno A, Iyer B, Ochsner E, Pan Y and Sathyaprakash B S 2009 arXiv:0907.0700

[16] Sathyaprakash B S and Dhurandhar S V 1991 Phys. Rev D 443819

[17] Abbott B et al (LIGO Scientific Collaboration) 2009 Phys. Rev. D 79122001 http://link.aps.org/abstract/ $\mathrm{PRD} / \mathrm{v} 79 / \mathrm{e} 122001$

[18] Abbott B P et al (LIGO Scientific) 2009 Phys. Rev. D 80047101 (arXiv:0905.3710)

[19] The LIGO Scientific Collaboration 2007 Tuning matched filter searches for compact binary coalescence Technical Report LIGO-T070109-01 http://www.ligo.caltech.edu/docs/T/T070109-01.pdf

[20] Abbott B et al (LIGO Scientific Collaboration) 2008 Phys. Rev. D 77062002 (arXiv:0704.3368) 
[21] Allen B, Anderson W G, Brady P R, Brown D A and Creighton J D E 2005 FINDCHIRP: an algorithm for detection of gravitational waves from inspiraling compact binaries arXiv:gr-qc/0509116

[22] Cokelaer T 2007 Phys. Rev. D 76102004 (arXiv:0706.4437)

[23] Robinson C A K, Sathyaprakash B S and Sengupta A S 2008 Phys. Rev. D 78062002

[24] Buonanno A and Damour T 1999 Phys. Rev. D 59084006

[25] Buonanno A and Damour T 2000 Phys. Rev. D 62064015

[26] Buonanno A et al 2007 Phys. Rev. D 76104049 (arXiv:0706.3732)

[27] Damour T and Nagar A 2008 Phys. Rev. D 77024043 (arXiv:0711.2628)

[28] Acernese F et al 2006 Class. Quantum Grav. 23 S187-S196 